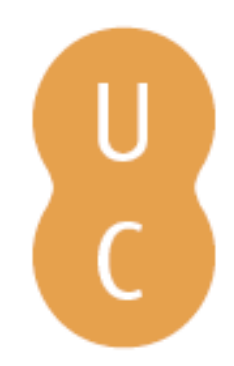

\title{
pombalina
}

\section{Escala do Impacto da Dor na Família (FIPS)}

Autor(es): $\quad$ Branco, Sandra; Portugal, Alda; Sotero, Luciana; Relvas, Ana Paula

Publicado por: Imprensa da Universidade de Coimbra

URL

persistente: URI:http://hdl.handle.net/10316.2/40592

DOI: $\quad$ DOl:https://doi.org/10.14195/978-989-26-1268-3_8

Accessed : $\quad$ 26-Apr-2023 15:22:03

A navegação consulta e descarregamento dos títulos inseridos nas Bibliotecas Digitais UC Digitalis, UC Pombalina e UC Impactum, pressupõem a aceitação plena e sem reservas dos Termos e Condições de Uso destas Bibliotecas Digitais, disponíveis em https://digitalis.uc.pt/pt-pt/termos.

Conforme exposto nos referidos Termos e Condições de Uso, o descarregamento de títulos de acesso restrito requer uma licença válida de autorização devendo o utilizador aceder ao(s) documento(s) a partir de um endereço de IP da instituição detentora da supramencionada licença.

Ao utilizador é apenas permitido o descarregamento para uso pessoal, pelo que o emprego do(s) título(s) descarregado(s) para outro fim, designadamente comercial, carece de autorização do respetivo autor ou editor da obra.

Na medida em que todas as obras da UC Digitalis se encontram protegidas pelo Código do Direito de Autor e Direitos Conexos e demais legislação aplicável, toda a cópia, parcial ou total, deste documento, nos casos em que é legalmente admitida, deverá conter ou fazer-se acompanhar por este aviso. 
AVALIAÇÃO

$$
\text { FAMILIAR Vorstil Aorracicio }
$$

Q)



$-1000000$

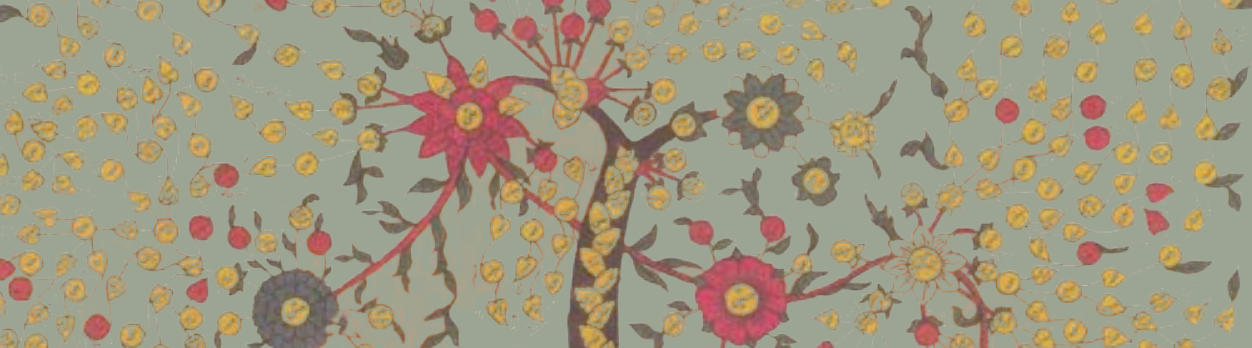

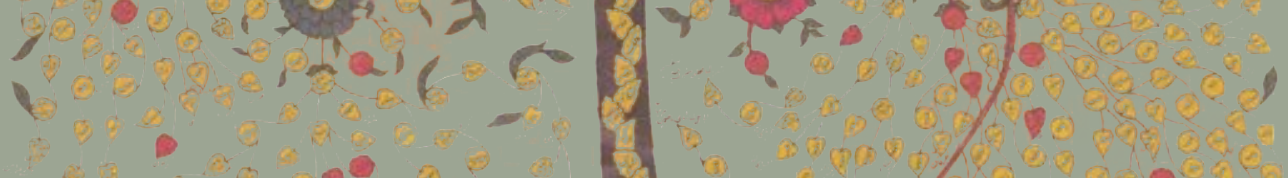

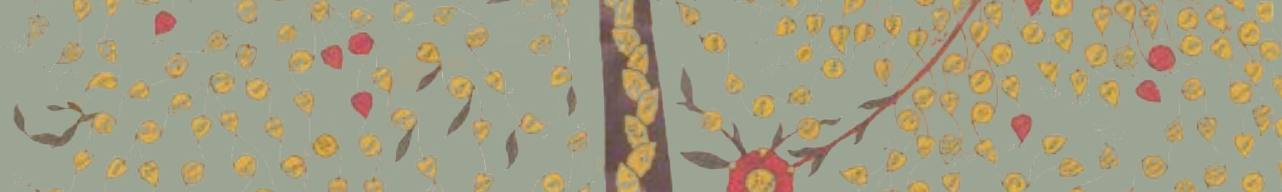

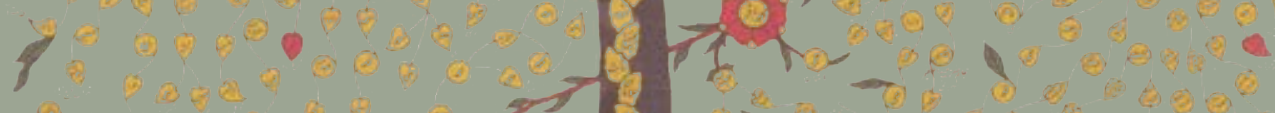

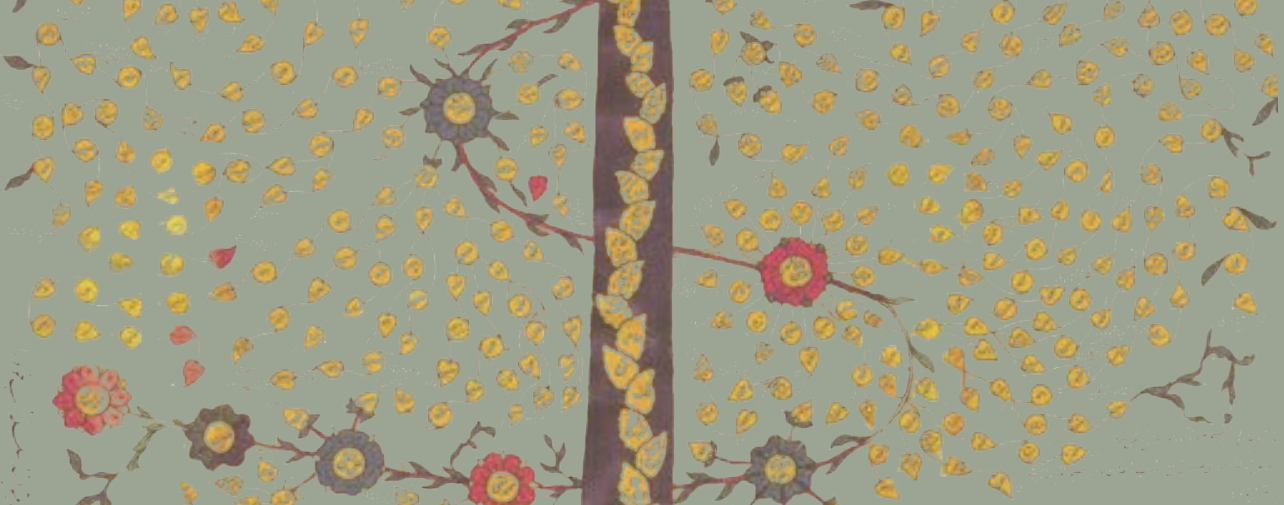

ANA PAULA RELVAS

SOFFA MAJOŔR Wh DE COIMBRA

COORDENAÇÃO 


\title{
ESCALA DO IMPACTO DA DOR NA FAMÍLA ( F I P S )
}

\author{
Sandra Branco \\ Alda Portugal \\ Luciana Sotero \\ Ana Paula Relvas
}

"Um dos aspetos mais fascinantes da dor é a ambiguidade da sua presença entre o corpo e a mente."

(Lima \& Trad, 2007, p. 2672)

\section{Resumo}

A Escala do Impacto da Dor na Família é a versão portuguesa da The Family Impact of Pain Scale (FIPS; Newton-John, 2005), um questionário de auto-resposta composto por 10 itens que permite avaliar o impacto da dor crónica na família. A partir de uma amostra de 51 sujeitos com dor crónica foram realizados estudos de tradução e adaptação para a população portuguesa, assim como estudos descritivos, de precisão e de validade de constructo. Os resultados demonstram uma elevada consistência interna dos itens $(\alpha=.91)$. Na análise fatorial confirmatória não se verificou uma correspondência com os fatores da escala original. As correlações, com base no coeficiente de Spearman, permitem concluir que existe uma relação estatisticamente significativa entre o impacto da dor crónica na família e as crenças de autoeficácia e a depressão 
( $r=-.754, p<.01 ; r=.332, p<.05$, respetivamente). Futuramente, o estudo da dor crónica na família deverá contemplar, não só os sujeitos com dor crónica, como também os seus familiares.

Palavras-chave: dor crónica, família, FIPS, estudos psicométricos.

\begin{abstract}
The Escala do Impacto da Dor Crónica na Família is the Portuguese version of The Family Impact of Pain Scale (FIPS; Newton-John, 2005), a 10-item self-report scale assessing the impact of chronic pain in the family. Based on a sample of 51 subjects with chronic pain, studies of translation and adaptation for the Portuguese population were performed, as well as descriptive, reliability, and construct validity studies. The results reveal strong reliability for the items $(\alpha=.91)$. In confirmatory factor analysis it wasn't found a correspondence with the factor structure of the original scale. Correlations based on Spearman's coefficient allow us to conclude that there is a statistical significant relationship between the impact of chronic pain in the family and the self-efficacy beliefs and depression $(r=-.754, p<.01 ; r=.332$, $p<.05$, respectively). In the future, the study of chronic pain in the family should include not only subjects with chronic pain as well as their relatives.
\end{abstract}

Keywords: chronic pain, family, FIPS, psychometric studies.

\title{
1. Instrumento
}

O que é, o que avalia e a quem se aplica?

No Quadro 1 apresenta-se a ficha técnica da versão original da Escala do Impacto da Dor na Família (FIPS; Newton-John, 2005). 
Quadro 1.

Ficha técnica da FIPS

\section{O que é?}

A Escala do Impacto da Dor na Família é versão portuguesa da The Family Impact of Pain Scale (FIPS), publicado originalmente em 2005 por Toby Newton-John, em Inglaterra

A FIPS é uma escala de auto-resposta composta por 10 itens que pretende avaliar em que medida as atividades e interações familiares (e.g., "levar a cabo tarefas domésticas", "ter uma vida social com a família") são afetadas na presença de dor crónica num dos seus elementos. Os 10 itens da escala encontram-se repartidos por duas subescalas: Atividade Física e Interação Pessoal (versão original)

\begin{tabular}{lcl}
\hline & Estrutura da FIPS (versão original) \\
\hline Subescala & Número Itens $\quad$ Descrição
\end{tabular}

Mede a forma como as limitações físicas

\section{O que avalia?}

Atividade Física

(AF)
8 decorrentes da dor crónica interferem na execução de determinadas atividades com algum grau de esforço físico [e.g., item 1 - "Levar a cabo tarefas domésticas (por ex. fazer compras, limpeza, etc.)"]

Avalia em que medida a dor crónica afeta a Interação Pessoal (IP)
2 realização de atividades de interação interpessoal (e.g., item 5 - "Estar envolvido em decisões familiares")

A FIPS foi desenvolvida para compreender a perceção do sujeito que padece de dor crónica relativamente ao impacto da sua dor no seio

A quem se aplica? familiar, sendo, assim, aplicada a sujeitos com dor crónica e idade igual ou superior a 18 anos. A escala destina-se à utilização em contexto clínico e de investigação

O acesso à FIPS pode ser efetuado através da página http://www.fpce. uc.pt/avaliaçaofamiliar que contém todos os instrumentos de avaliação apresentados neste livro. Os utilizadores deverão facultar os contactos

Como ter acesso? pessoais e institucionais, bem como dados acerca do propósito da utilização da FIPS (e.g. investigação, prática clínica) e concordar com as condições de utilização e de partilha dos resultados com os autores da versão portuguesa

\title{
Fundamentação e história
}

\author{
A experiência de dor é um fenómeno complexo e subjetivo e, por \\ isso, difícil de definir, no sentido em que a sua perceção e interpretação \\ difere de pessoa para pessoa (Dias, 2007; Melzack, 1996; Silva, Neto,
}


Figueiredo, \& Barbosa-Branco, 2007; Silver, 2004), estando relacionada com as idiossincrasias de quem a experiencia (Silva et al., 2007). Deste modo, na literatura são apresentadas diversas definições de dor crónica. A International Association for the Study of Pain (IASP) (citado por Azevedo, Costa-Pereira, Mendonça, Dias, \& Castro-Lopes, 2012) define dor crónica como uma dor que permanece durante três meses ou mais, após o tempo normal de cicatrização dos tecidos da lesão que lhe deram origem. Relativamente à duração da dor, a IASP considera conveniente um ponto de corte de três meses (Azevedo et al., 2012), contudo este critério temporal não é consensual entre os profissionais de saúde. Em Portugal, tal como contemplado no Plano Nacional de Controlo da Dor (2008), a Direção Geral da Saúde tem por base a definição proposta pela IASP. Ainda assim, o período mínimo de tempo de seis meses parece ser o mais usual (Silver, 2004).

Também o Manual de Diagnóstico e Estatística das Perturbações Mentais (DSM-IV-TR; American Psychiatric Association, 2002) descreve a dor como uma condição crónica de doença. Segundo os critérios do DSM-IV-TR, a dor crónica, com duração igual ou superior a seis meses, pressupõe:

a) a existência de dor anatómica, numa ou mais localizações, cuja gravidade é suficiente para merecer atenção clínica; b) sofrimento clinicamente significativo e incapacidade social, ocupacional ou noutras áreas importantes do funcionamento individual; c) que os fatores psicológicos desempenhem um papel importante no início, gravidade, exacerbação e manutenção da dor; d) que o sintoma ou défice não seja intencional ou simulado; e e) que a dor não seja explicada por uma perturbação do humor, da ansiedade ou psicótica e não preencha os critérios de dispareunia.

Estudos epidemiológicos relatam uma elevada prevalência da dor crónica na Europa. Uma investigação realizada por Breivik, Collett, Ventafridda, Cohen e Gallacher (2006) em 15 países europeus (nos quais Portugal não está incluído) e em Israel permitiu concluir que a dor crónica afeta $19 \%$ dos adultos. Na população portuguesa, no estudo levado a cabo por Azevedo e colaboradores (2012), concluiu-se que cerca de 36,7\% da população adulta portuguesa sofre de dor crónica. 
No que diz respeito às condições de dor mais comuns, Silver (2004) identifica a artrite, dor lombar, dor facial, dor de cabeça, fibromialgia, dor de pescoço, dor abdominal, dor pélvica e dor no pé. Atendendo a estes indicadores e às descrições da literatura sobre o impacto negativo a nível pessoal, familiar e social (Dias, 2007; Lima \& Trad, 2007; Miceli, 2002; Seymour \& Paz, 2004; West, Usher, Foster, \& Stewart, 2012), torna-se evidente a pertinência do estudo do impacto da dor crónica no doente e seu sistema familiar.

Nos últimos anos, a investigação no âmbito dor crónica tem-se debruçado sobre o impacto desta problemática a nível individual, ou seja, nos efeitos diretos sobre a vida dos doentes. A literatura identifica dificuldades na expressão de emoções e na capacidade de envolvimento empático entre os pacientes e os restantes membros da família (Roy, 2006), o distress emocional e consequente distanciamento do sujeito em relação à família (Smith \& Friedemann, 1999), o impacto ao nível emocional com sentimentos de autoculpabilização, de raiva e medo (West, Usher, et al., 2012) e, de um modo geral, dificuldades na realização de tarefas domésticas e ocupacionais (Roy, 2006; Silver, 2004; Smith \& Friedemann, 1999; West, Usher, et al., 2012).

Por sua vez, o impacto da dor crónica no seio da família traduz-se, habitualmente, em dificuldades em diversas dimensões do funcionamento familiar, tais como a resolução de problemas familiares, a relação conjugal, a (re)definição de papéis e a comunicação (Roy, 2006; Silver, 2004; Smith \& Friedemann, 1999; West, Usher, et al., 2012). Prevê-se que ocorram alterações na estrutura e dinâmicas familiares, dado que, frequentemente, as relações e funções familiares se concentram nos cuidados prestados ao familiar doente (Bazako, 2003; Lopes, 2007; Martins, 2009; Power \& Orto, 2004, citados por Fernandes, 2011), tendo em consideração que "a dor crónica é acompanhada por um conjunto complexo de alterações somáticas e psicossociais" (Coniam \& Diamond, 2001 citado por Dias, 2007 , p. 2), Contudo, este processo não é linear, pelo que varia de família para família, dependendo de fatores como os recursos (e.g., emocionais e financeiros) que os sujeitos possuem, a adaptação e a coesão familiar (Fernandes, 2011). 
Apesar da vasta investigação descrita anteriormente, a falta de instrumentos de avaliação do impacto da dor crónica na família representa uma lacuna nesta área. Alguns dos estudos mencionados recorrem a instrumentos de avaliação diádicos, tais como o Lock-Wallace Marital Adjustment (Locke \& Wallace, 1959) ou a escala Dyadic Adjustment (Spanier, 1976), sendo que a perspetiva familiar parecia estar a descoberto. Estes instrumentos avaliam de forma limitada o impacto da dor na família, pois destinam-se apenas a avaliar o efeito da dor na díade conjugal, ou seja, as perceções dos restantes membros da família não são tidas em conta no momento da avaliação (Newton-John, 2005). Neste sentido, a The Family Impact of Pain Scale foi desenvolvida com o objetivo de avaliar o impacto da dor crónica nas atividades e interações familiares, tendo, assim, a mais-valia de considerar todos os elementos da família, possibilitando uma melhor compreensão do impacto da dor crónica na família, englobando todos os sujeitos relacionados biológica, emocional ou legalmente (West, Beuttner, Foster, \& Usher, 2012).

$\mathrm{Na}$ investigação original da FIPS (Newton-John, 2005), os itens da escala derivaram de outros instrumentos de avaliação de incapacidade e da observação clínica. Inicialmente, o grupo de investigadores desenvolveu uma escala de 18 itens que foi alvo de um estudo-piloto, por forma a assegurar a legibilidade e a adaptabilidade para a maioria dos padrões familiares. Deste estudo inicial resultaram os 10 itens da FIPS, tendo sido, posteriormente, utilizadas duas amostras para estudar as suas qualidades psicométricas. Aos participantes era pedido que refletissem sobre cada item e, posteriormente, indicassem, numa escala de 0 a 10 , em que 0 corresponde a "a dor não interfere" e 10 a "a dor interfere completamente", em que medida a dor teria impacto na execução de cada atividade. A primeira amostra foi constituída por 113 sujeitos que sofriam de dor há pelo menos seis meses, sem recorrer a cirurgia ou a outros tratamentos invasivos, ou seja, sem qualquer tipo de intervenção médica relativa ao tratamento ou controlo da dor. A estes sujeitos foi administrado um protocolo de avaliação com o intuito de avaliar o impacto da dor crónica na família, a intensidade da dor, a sintomatologia depressiva, 
a utilização de estratégias de coping, a autoeficácia e as capacidades físicas. A segunda amostra incluía 64 pacientes com dor crónica (critérios de inclusão de duração da dor semelhantes aos da primeira amostra) que frequentavam um programa de controlo da dor, de orientação cognitivo-comportamental, no Hospital Nacional de Neurologia e Neurocirurgia de Londres. Estes sujeitos participaram num programa de controlo da dor em nove tratamentos durante oito semanas (dois dias na primeira semana) e completaram a bateria de instrumentos de avaliação e uma série de avaliações físicas no primeiro e no último dia do programa. Posteriormente, foram realizadas sessões de follow-up após 1, 3, 6, e 12 meses. Para estudar a consistência interna, a estrutura fatorial, a validade convergente e divergente e a validade critério foram utilizados os dados da primeira amostra. A segunda amostra foi recolhida com o propósito de explorar a sensibilidade clínica da escala, isto é, a sua capacidade para detetar os efeitos dos tratamentos.

Os estudos efetuados indicam que a FIPS surge como uma ferramenta adequada para avaliar o impacto da dor crónica na qualidade de vida do sujeito e da sua família, salientando-se diversas vantagens na sua utilização, nomeadamente, o facto de ser um questionário breve, fácil de administrar e aplicável a indivíduos com dor crónica que tenham uma variedade de pessoas significativas na sua vida (e.g., companheiro, pais, irmãos, filhos, colegas) (Newton-John, 2005). O autor concluiu, também, que a escala possui uma boa estabilidade temporal, elevada consistência interna $(\alpha=.94)$ e validade preditiva e de constructo, evidenciando a fiabilidade dos resultados obtidos com a escala. Relativamente à estrutura fatorial, a escala original apresentou um modelo bifatorial, com uma dimensão relativa à Atividade Física (constituída pelos itens 1, 2, 4, 6, 7, 8, 9 e 10) e uma dimensão referente à Interação Pessoal (itens 3 e 5). No que diz respeito à intervenção cognitivo-comportamental no controlo da dor ( $2^{\mathrm{a}}$ amostra), os resultados da investigação revelam uma sensibilidade adequada, ou seja, a escala possibilita uma boa compreensão dos efeitos da intervenção no controlo da dor, dado que os sujeitos apresentaram resultados mais baixos nas escalas aplicadas após o programa de intervenção. 
Para além dos estudos já reportados, a respeito da escala original, existe ainda a aferição da FIPS para a população Australiana. Esta última foi levada a cabo por West, Buettner e colaboradores (2012), com uma amostra de 67 pacientes com dor crónica em North Queensland, Austrália. Nesta investigação, tanto os sujeitos com dor crónica $(n=31)$, como os respetivos familiares $(n=36)$ responderam à FIPS. Foi também elaborado um protocolo de avaliação que incluía, para além da FIPS, escalas de avaliação da resiliência, do estado de saúde geral físico e mental e da perceção da disponibilidade de apoio funcional. Conforme referido, nesta investigação foram avaliadas as perceções dos sujeitos com dor e, também, dos seus familiares, permitindo concluir que os indivíduos que padecem de dor consideram existir um impacto médio da dor na família (FIPS: $M=5.25 ; D P=2.22$ ), ao passo que os outros elementos da família revelam um menor impacto (FIPS: $M=4.39 ; D P=2.25$ ). Relativamente às qualidades psicométricas, os resultados revelaram-se consistentes com o estudo anterior, indicando elevada consistência interna $(\alpha=.89)$ e validade preditiva e de constructo, demonstrando que a FIPS é uma medida fiável do impacto da dor crónica nas famílias australianas (West, Beuttner, et al., 2012).

Deste modo, tanto na população inglesa como na australiana, pode concluir-se que a FIPS possui uma elevada consistência interna, sendo uma medida fiável do impacto da dor crónica na família.

\section{Estudos em Portugal \\ Como foi desenvolvido e adaptado?}

\section{Estudos de tradução e adaptação}

Inicialmente, o autor original da escala foi contactado, com vista ao pedido de permissão para a utilização da mesma para fins de investigação e realização de estudos psicométricos. Após uma resposta afirmativa por parte do autor, deu-se início ao processo de tradução 
para português que, de acordo com as diretrizes da Organização Mundial de Saúde (OMS; s.d.), passou por quatro fases distintas: a) tradução do instrumento (inglês-português) realizada por um indivíduo cuja língua materna é o português; b) revisão da primeira tradução (inglês-português), efetuada por um indivíduo bilingue no sentido de corrigir e adaptar expressões inadequadas para a língua portuguesa; c) retroversão realizada por um tradutor independente, ou seja, a versão portuguesa, resultante da segunda fase foi traduzida novamente para o inglês; e, d) finalmente, realização de um estudo-piloto, de modo a testar a tradução portuguesa e avaliar, por parte dos respondentes, a validade facial do instrumento, isto é, a compreensão das instruções, dos itens e dos níveis de resposta. Nesta última fase, a escala foi aplicada a seis sujeitos com dor crónica tendo sido realizada uma entrevista com as seguintes questões: a) "O que pensa dessa questão?"; b) "Consegue repeti-la por palavras suas?”; c) "Consegue explicar como escolheu a sua resposta?"; e d) "Existe alguma palavra ou expressão que não tenha compreendido ou que considere de algum modo ofensiva ou inaceitável?". As respostas dos participantes indicaram a necessidade de proceder a um ajustamento no item 2 "Participar em atividades de lazer". Quatro dos seis inquiridos revelaram não compreender a palavra "lazer" e, por esse motivo, decidiu-se acrescentar um exemplo "atividades praticadas nos tempos livres".

Elaborada a versão final do instrumento, iniciou-se a divulgação do estudo (presencialmente e/ou via $e$-mail) em diversos serviços de saúde, explicando a problemática, os objetivos e os critérios de inclusão. Desta fase de divulgação, foi possível obter a autorização, para recolha de amostra, do Centro de Saúde Norton de Matos do Agrupamento de Centros de Saúde ACES do Baixo Mondego. Seguiu-se a aplicação do protocolo, o qual incluía o questionário de dados sociodemográficos, a Escala de Ansiedade e Depressão Hospitalar (HADS; Pais-Ribeiro et al., 2007), a Portuguese Version of the Pain Self-Efficacy Questionnaire (P-PSEQ; Ferreira-Valente, Pais-Ribeiro, \& Jensen, 2011) e a Escala do Impacto da Dor na Família (versão portuguesa da FIPS), preenchidos através do método tradicional de papel 
e lápis. O questionário sociodemográfico incluía questões relacionadas com a duração da dor crónica, a patologia associada, o(s) local(ais), a intensidade e os sintomas ou alterações devido à dor. A HADS tem como principal objetivo auxiliar o profissional de saúde a identificar as componentes emocionais associadas às doenças físicas. Trata-se de uma escala bidimensional composta pelas subescalas de ansiedade e depressão, cada uma delas constituída por sete itens (Pais-Ribeiro et al., 2007). A versão portuguesa do Pain Self-Efficacy Questionnaire é um questionário de auto-resposta que avalia a confiança de sujeitos que padecem de dor crónica na realização de diversas atividades quotidianas, nomeadamente tarefas domésticas, atividades sociais e trabalho (Ferreira-Valente et al., 2011). Neste sentido, indo de encontro à literatura e tentando replicar o estudo original da FIPS, tentou-se compreender se existe relação entre a ansiedade e depressão, a autoeficácia e o impacto da dor crónica no seio familiar.

O processo de recolha da amostra foi baseado no método não-probabilístico de bola de neve, ou seja, foi gerada uma rede de contactos a partir da aplicação do protocolo aos primeiros pacientes (Pais-Ribeiro, 2007). Previamente à aplicação do protocolo, foi dada a conhecer a todos os participantes a investigação e seguiram-se os princípios éticos, a natureza anónima e confidencial do estudo (APA, 2010).

Os critérios de inclusão utilizados foram: a) sujeitos com idade igual ou superior a 18 anos e b) diagnóstico de dor crónica há pelo menos três meses, tendo por base a definição de dor crónica proposta pela IASP (citado por Azevedo et al., 2012) e, também, considerada pela Direção Geral da Saúde.

A amostra recolhida abrange um total de 51 sujeitos com idades compreendidas entre os 18 e os 86 anos $(M=51.94 ; D P=21.75)$, dos quais 40 são mulheres (78.4\%) e 11 são homens (21.6\%), com diferentes níveis de escolaridade (desde o analfabetismo até ao mestrado). Relativamente ao estado civil, $52.9 \%$ dos sujeitos são casados ou vivem em união de facto e $21.6 \%$ são solteiros. Quanto à situação profissional, $39.3 \%$ dos sujeitos estão atualmente empregados. Para obter a classificação do nível socioeconómico (NSE), baseamo-nos na 
proposta de Simões (2000), no qual verificamos que a categoria mais representativa é a classe média (56.9\%) (cf. Quadro 2).

Quadro 2.

Caracterização da amostra

\begin{tabular}{|c|c|c|c|}
\hline Variáveis & & $n$ & $\%$ \\
\hline \multirow[t]{5}{*}{ Sexo } & Masculino & 11 & 21.6 \\
\hline & Feminino & 40 & 78.4 \\
\hline & 18-27 anos & 10 & 19.6 \\
\hline & 28-37 anos & 4 & 7.8 \\
\hline & $38-47$ anos & 9 & 17.6 \\
\hline \multirow[t]{6}{*}{ Idade } & $48-57$ anos & 9 & 17.6 \\
\hline & 58-67 anos & 4 & 7.8 \\
\hline & 68-77 anos & 6 & 11.8 \\
\hline & 78-87 anos & 9 & 17.6 \\
\hline & $1^{\circ}$ Ciclo $\left(0-4^{\circ}\right.$ ano $)$ & 19 & 37.3 \\
\hline & $2^{\circ}$ Ciclo $\left(5^{\circ}-6^{\circ}\right.$ ano $)$ & 1 & 2.0 \\
\hline \multirow{3}{*}{ Escolaridade } & $3^{\circ}$ Ciclo $\left(7^{\circ}-9^{\circ}\right.$ ano $)$ & 10 & 19.6 \\
\hline & Ensino Secundário $\left(10^{\circ}-12^{\circ}\right.$ ano $)$ & 12 & 23.5 \\
\hline & $\begin{array}{l}\text { Ensino Superior (Bacharelato, } \\
\text { Licenciatura ou Mestrado) }\end{array}$ & 7 & 13.7 \\
\hline \multirow{5}{*}{ Estado Civil } & Solteiro & 11 & 21.6 \\
\hline & Casado/União de facto & 27 & 52.9 \\
\hline & Divorciado/Separado & 8 & 15.7 \\
\hline & Viúvo & 5 & 9.8 \\
\hline & Baixo & 15 & 29.4 \\
\hline \multirow[t]{2}{*}{ NSE } & Médio & 29 & 56.9 \\
\hline & Elevado & 6 & 11.8 \\
\hline
\end{tabular}

A duração média de anos de dor crónica corresponde a 9.60 anos ( $D P$ = 9.46; mín $=3$ meses, máx $=38$ anos). De entre as condições de dor crónica mais comuns encontram-se a cefaleia (33.3\%), a lesão traumática (19.6\%) e a artrite reumatoide (15.7\%). Os locais do corpo com dor mais reportados são o tronco (47.1\%), a cabeça (33.3\%) e as pernas (33.3\%). No que respeita à intensidade da dor, obteve-se um resultado médio de 3.63, numa escala de 0 a $5(D P=0.77)$, sendo que sem dor corresponde a 0 , dor mínima a 1 , dor ligeira a 2 , dor moderada a 3 , dor intensa a 4 e dor máxima a 5. Os sintomas ou alterações associados à dor, mais referidos pelos sujeitos são as dificuldades de movimento (64.7\%), os problemas de sono (58.8\%), a fadiga (54.9\%) e a irritabilidade (49.0\%) (cf. Quadro 3). 
Quadro 3.

Caracterização da dor crónica

\begin{tabular}{|c|c|c|c|}
\hline Variáveis & & $n$ & $\%$ \\
\hline \multirow{8}{*}{$\begin{array}{l}\text { Tempo de diagnóstico da } \\
\text { dor crónica }\end{array}$} & $0-3$ meses & 1 & 2.0 \\
\hline & 4-6 meses & 1 & 2.0 \\
\hline & 7 meses- 1 ano & 3 & 5.9 \\
\hline & $2-4$ anos & 13 & 25.5 \\
\hline & 5-7 anos & 12 & 23.5 \\
\hline & 8-10 anos & 5 & 9.8 \\
\hline & 11-14 anos & 3 & 5.9 \\
\hline & $>15$ anos & 11 & 21.6 \\
\hline \multirow{8}{*}{$\begin{array}{l}\text { Patologia associada à dor } \\
\text { crónica }\end{array}$} & Cefaleia & 17 & 33.3 \\
\hline & Lombalgia & 3 & 5.9 \\
\hline & Artrite Reumatoide & 8 & 15.7 \\
\hline & Neuropatia & 1 & 2.0 \\
\hline & Osteoartrite & 2 & 3.9 \\
\hline & Lesão Traumática & 10 & 19.6 \\
\hline & Reabilitação Pós-cirúrgica & 2 & 3.9 \\
\hline & Outra $^{1}$ & 17 & 33.3 \\
\hline \multirow{8}{*}{ Local da dor } & Cabeça & 17 & 33.3 \\
\hline & Pescoço & 7 & 13.7 \\
\hline & Tronco & 24 & 47.1 \\
\hline & Braços & 10 & 19.6 \\
\hline & Mãos & 11 & 21.6 \\
\hline & Órgãos genitais & 1 & 2.0 \\
\hline & Pernas & 17 & 33.3 \\
\hline & Pés & 6 & 11.8 \\
\hline \multirow{4}{*}{ Intensidade da dor } & Dor ligeira & 2 & 3.9 \\
\hline & Dor moderada & 22 & 43.1 \\
\hline & Dor intensa & 20 & 39.2 \\
\hline & Dor máxima & 7 & 13.7 \\
\hline
\end{tabular}

${ }^{1}$ Outra $=$ Artrose, hérnia, fibromialgia, trombofilia, etc.

Estudos de análise de itens: Estatísticas descritivas

A análise descritiva dos itens 10 itens da FIPS encontra-se no Quadro 4, onde estão apresentados os valores da média, desvio-padrão e valores mínimos e máximos. Os resultados indicam que as atividades familiares que surgem mais comprometidas devido à dor são as tarefas 
domésticas, as atividades de lazer e a realização de planos para o futuro a longo-prazo e a curto-prazo. Relativamente à escala total obteve-se uma média de 4.05 ( $D P=2.24)$, numa escala de 0 a 10 , o que sugere um impacto médio da dor crónica na família na amostra recolhida. De um modo geral, os valores obtidos oscilam entre 0 e 10 (cf. Quadro 4).

Quadro 4.

Análise descritiva dos itens da FIPS

\begin{tabular}{lccc}
\hline Item & $M$ & $D P$ & Mín-Máx \\
\hline 1. Tarefas domésticas & 5.36 & 2.52 & $0-9$ \\
2. Atividades de lazer & 5.27 & 2.63 & $0-10$ \\
3. Comunicação com a família & 3.37 & 2.72 & $0-8$ \\
4. Fazer planos - curto-prazo & 4.08 & 2.77 & $0-10$ \\
5. Envolvimento em decisões familiares & 3.14 & 2.84 & $0-10$ \\
6. Fazer planos - longo-prazo & 4.14 & 3.18 & $0-10$ \\
7. Assumir responsabilidades familiares & 3.88 & 2.98 & $0-10$ \\
8. Socializar com a família & 3.35 & 2.74 & $0-8$ \\
9. Cuidar de filhos/crianças & 3.00 & 2.81 & $0-9$ \\
10. Relação física com o parceiro & 3.78 & 3.37 & $0-10$ \\
\hline
\end{tabular}

\section{Estudos de precisão}

A fiabilidade dos itens da FIPS foi estudada através da análise da consistência interna, pelo cálculo do coeficiente alfa de Cronbach. O valor do alfa de Cronbach do resultado total da FIPS foi de .91, o que demonstra forte consistência interna (Pallant, 2005), isto é, a escala constitui uma medida fiável do estudo do impacto da dor crónica na família. Na versão original da FIPS e na versão validada para a população australiana, os valores do alfa de Cronbach foram de .94 e .89, respetivamente.

A análise dos valores do coeficiente alfa da escala total, aquando da exclusão de qualquer um dos itens, indica-nos que a sua exclusão 
não aumenta de forma expressiva a consistência interna total da escala. Os valores de correlação item-total revelam uma adequada capacidade discriminante de todos os itens ( $r>.30)$ (Wilmut, 1975) (cf. Quadro 5).

Quadro 5.

Estatísticas da correlação item-total e do alfa com eliminação do item

\begin{tabular}{lcc}
\hline Item & Correlação Item-Total Corrigida & Alfa com Item Excluído \\
\hline 1 & .53 & .91 \\
2 & .62 & .91 \\
3 & .77 & .90 \\
4 & .60 & .91 \\
5 & .85 & .90 \\
6 & .61 & .91 \\
7 & .67 & .90 \\
8 & .92 & .89 \\
9 & .70 & .90 \\
10 & .64 & .91 \\
\hline
\end{tabular}

Estudos de validade de constructo: Análise fatorial confirmatória (AFC)

A validade interna da versão portuguesa da FIPS foi verificada através da análise fatorial confirmatória (AFC) recorrendo ao software AMOS 22. Realizámos assim uma AFC do modelo bifatorial da escala original da FIPS: F1 (Atividade Física - itens 1, 2, 4, 6, 7, 8, 9 e 10) e F2 (Interação Pessoal - itens 3 e 5).

A análise dos dados indica que a estrutura bifatorial original apresenta índices de ajustamento desadequados para a amostra em estudo. Os resultados da AFC indicaram um qui-quadrado absoluto $\left(\chi^{2}\right)$ de 59.482 $(p=.004)$, um qui-quadrado normalizado $\left(\chi^{2} / g l\right)$ de 1.749 , sendo o valor do Comparative Fit Index de .91. No que diz respeito à raiz quadrada da média do erro de aproximação (RMSEA), o valor foi de .12. Considerando os valores critério de referência $\left[x^{2} / g l<5\right.$; CFI $>$.95; RMSEA $\left.<.05\right]$ propostos por Marôco (2010), conclui-se que os resultados não se ajustam à estrutura original da FIPS proposta pela investigação de Newton-John (2005). De notar que não foram realizados reajustes na impossibilidade 
de calcular os índices de modificação devido à existência de missings na amostra utilizada. Neste sentido, optou-se por considerar os 10 itens num único fator dado que a estrutura bifatorial não se ajustou.

\section{Estudos de validade de constructo - Correlações entre variáveis}

De modo a analisar a validade de constructo da escala procedeu-se ao estudo das correlações entre os resultados das duas dimensões da HADS, do P-PSEQ, da intensidade da dor, da idade e o resultado total da FIPS, através do cálculo dos coeficientes de Spearman. Os resultados indicaram que a relação entre a dimensão Ansiedade da HADS e a FIPS apresenta uma correlação de $.15(p=.300)$, estatisticamente não significativa. No que diz respeito à relação da dimensão Depressão da HADS e a FIPS, obteve-se uma correlação de $.33(p<.05)$, indicando uma relação positiva moderada. Isto é, quanto maior o impacto da dor na família, maior será a depressão do paciente com dor crónica. Relativamente à associação entre o P-PSEQ e a FIPS, através de um coeficiente de correlação de $-.75(p<.01)$, conclui-se que existe uma relação negativa forte, o que sugere que quanto menor for o impacto da dor na família, maiores são as crenças de autoeficácia do sujeito com dor. A intensidade da dor e a FIPS é representada por um coeficiente de correlação de $.22(p=.114)$, estatisticamente não significativo. Por último, estabeleceu-se a relação entre a variável idade e a FIPS, tendo obtido um coeficiente de correlação de .068 ( $p=.633)$, estatisticamente não significativo (cf. Quadro 6).

Quadro 6.

Correlações entre a FIPS e a HADS, o P-PSEQ, a intensidade da dor e a idade

\begin{tabular}{lll}
\hline Variáveis & FIPS \\
\hline HADS & Ansiedade & .148 \\
& Depressão & $.332^{*}$ \\
P-PSEQ & $-.754^{* *}$ \\
Intensidade da dor & .224 \\
Idade & .068 \\
\hline
\end{tabular}

Nota: HADS - Escala de Ansiedade e Depressão Hospitalar; P-PSEQ - Portuguese Version of the Pain Self-Efficacy Questionnaire.

$* p<.05 \quad * * p<.01$ 


\section{Aplicação}

\section{Como aplicar, cotar e interpretar?}

O material necessário para a aplicação da escala é apenas a versão em papel e uma caneta. A versão portuguesa da FIPS pretende avaliar em que medida as atividades e interações familiares são afetadas na presença de dor crónica num dos seus elementos, de modo a compreender o ponto de vista dos pacientes com dor crónica. Pretende-se que o respondente reflita sobre cada um dos 10 itens e indique, numa escala de 0 a 10, em que 0 corresponde a "a dor não interfere" e 10 a "a dor interfere completamente", em que medida a dor tem impacto em cada uma das atividades referidas no questionário. Os resultados da FIPS advêm da soma das pontuações do total dos itens da escala e divisão pelo número de itens. Neste sentido, resultados elevados indicam dificuldade na gestão familiar da dor.

\section{Vantagens, limitações e estudos futuros}

A FIPS apresenta-se como uma escala breve, fácil de administrar e de cotar que permite compreender a perceção do impacto da dor crónica nos sujeitos e suas famílias (Newton-John, 2005). Tanto quanto é do nosso conhecimento, não existia em Portugal um instrumento de compreensão do impacto da dor na família que pudesse ser aplicado em contexto de avaliação, intervenção e investigação. Este facto revela a pertinência e importância do presente estudo, podendo este ser um ponto de partida para analisar e aprofundar a questão do impacto da dor crónica nas famílias portuguesas. Assim, este trabalho pretende auxiliar os profissionais de saúde na análise, compreensão e intervenção junto dos sujeitos que padecem de dor crónica e respetivas famílias, de modo a gerir de forma mais eficaz as exigências e alterações inerentes à dor crónica.

Apesar das boas qualidades psicométricas da versão portuguesa da FIPS, especificamente ao nível da consistência interna, o estudo apresenta algumas limitações relacionadas com a amostra. Nomeadamente, o facto 
de se tratar de uma amostragem por conveniência, pelo que a amostra recolhida poderá não ser representativa da população que pretendemos estudar. Estas limitações associam-se ainda com o tamanho reduzido da amostra (51 sujeitos) e com a sua fraca heterogeneidade em termos de sexo, uma vez que existe uma grande discrepância entre o número de homens e de mulheres. Limitações semelhantes a estas estão também presentes no estudo original ( $N=113,81$ mulheres e 32 homens) e no estudo Australiano ( $N=67,35$ mulheres e 32 homens). De notar, ainda, que se constitui uma limitação ao nível das qualidades psicométricas da versão portuguesa da FIPS, o facto da estrutura original da FIPS não se ajustar aos dados recolhidos.

Estudos futuros com a FIPS deverão incluir a perspetiva da família do paciente com dor, de modo a comparar as diferentes leituras, tornando mais rico o conhecimento sobre este tema ao permitir estabelecer comparações relativas à perceção de pacientes e familiares sobre o impacto da dor no seio familiar. Poder-se-á, também, estudar a FIPS no âmbito de populações específicas, formando diversos grupos com diferentes causas de dor (e.g., sujeitos com enxaquecas, fibromialgia, artrite reumatoide, lesões traumáticas, entre outros). Será ainda fundamental que, no futuro, possam ser realizados mais estudos de precisão e validade, no sentido de reforçar as propriedades psicométricas da FIPS.

\section{Bibliografia}

American Psychiatric Association (2002). DSM-IV-TR: Manual de diagnóstico e estatística das perturbações mentais ( $4 .^{a}$ ed., texto revisto, J. N. Almeida, trad.). Lisboa: Climepsi. (Trabalho original publicado em 2000).

American Psychological Association (2010). Ethical principles of psychologists and code of conduct. Acedido em http://www.apa.org/ethics/code/principles.pdf.

Azevedo, L. F., Costa-Pereira, A., Mendonça, L., Dias, C. C., \& Castro-Lopes, J. M. (2012). Epidemiology of chronic pain: A population-based nationwide study on its prevalence, characteristics and associated disability in Portugal. The Journal of Pain: Official Journal of the American Pain Society, 13(8), 773-783. doi:10.1016/j.jpain.2012.05.012

Bazako, E. (2003). Intervención psicológica en una unidade de dolor. In Remor, E, Arranz, P., \& Ulla, S. (Eds), El psicólogo en el ámbito hospitalário (pp. 569-590). Desclée De Brouwer: Bilbao. 
Breivik, H., Collett, B., Ventafridda, V., Cohen, R., \& Gallacher, D. (2006). Survey of chronic pain in Europe: Prevalence, impact on daily life, and treatment. European Journal of Pain, 10, 287-33. doi:10.1016/j.ejpain.2005.06.009

Dias, A. (2007). Dor Crónica - um Problema de Saúde Pública. Acedido em http://www. psicologia.pt/artigos/textos/A0372.pdf.

Direção Geral da Saúde (2008). Plano Nacional de Controlo da Dor. Circular Normativa N ${ }^{\circ}$ 11/DSCS/DPCD de 18/06.

Fernandes, D. (2011). Dor crónica: Adaptabilidade e coesão familiar dor crónica (Dissertação de Mestrado). Faculdade de Filosofia, Universidade Católica Portuguesa, Portugal. Acedido em http://repositorio.ucp.pt/bitstream/ 10400.14/8791/1/Tese\%20de\%20Mestrado\%20Final.pdf

Ferreira-Valente, A., Pais-Ribeiro, J., \& Jensen, M. P. (2011). Psychometric properties of Portuguese version of the pain self-efficacy questionnaire. Acta Portuguesa de Reumatologia, 1(6), 260-267.

Lima, M. A., \& Trad, L. A. (2007). A dor crônica sob o olhar médico: Modelo biomédico e prática clínica. Cadernos de Saúde Pública, 23(11), 2672-2680.

Locke, H. J., \& Wallace, K. M. (1959). Short marital adjustment and prediction tests: Their reliability and validity. Marriage and Family Living, 21, 251-255.

Lopes, A. (2007). Generalidades e singularidades da doença em família: Percepção da qualidade de vida, stress e coping. (Dissertação de Mestrado não publicada). Universidade de Coimbra, Coimbra, Portugal.

Marôco, J. (2010). Análise de equações estruturais: Fundamentos teóricos, software \& aplicações. Pêro Pinheiro: ReportNumber.

Martins, M. M. (2009). A consulta telefónica como intervenção de enfermagem ao doente/ família com dor crónica (Dissertação de Mestrado). Universidade Aberta, Lisboa, Portugal. Acedido em https://repositorioaberto.uab.pt/bitstream/10400.2/1473/1/Disserta \%C3\%A 7\%C3\%A30\%20\%20Mestrado\%20Comunica\%C3\%A7\%C3\%A30\%20 em\%20 Sa\%C3\%BAde\%20-\%20Madalena\%20Martins.pdf

Melzack, R. (1996). Gate control theory. Pain Forum, 5(2), 128-138. doi:10.1016/S10823174(96)80050-X.

Miceli, A. V. (2002). Dor crônica e subjetividade em oncologia. Revista Brasileira de Cancerologia, 48(3), 363-373.

Newton-John, T. R. (2005). The Family Impact of Pain Scale: Preliminary validation. Journal of Clinical Psychology in Medical Settings, 12(4), 349-358. doi:10.1007/s10880-005-7821-1

Organização Mundial de Saúde (s.d.). Process of translation and adaptation of instruments. Acedido em http://www.who.int/substance_abuse/ research_tools/translation/en/.

Pais-Ribeiro, J. (2007). Metodologia de investigação em psicologia e saúde. Porto: Legis.

Pais-Ribeiro, J., Silva, I., Ferreira, T., Martins, a, Meneses, R., \& Baltar, M. (2007). Validation study of a Portuguese version of the Hospital Anxiety and Depression Scale. Psychology, Health \& Medicine, 12(2), 225-235. doi:10.1080/13548500500524088

Pallant, J. (2005). SPSS survival manual, $\left(2^{\text {nd }}\right)$. Sydney: Allen\&Unwin.

Roy, R. (2006). Chronic pain and family: A clinical perspective. New York: Springer.

Seymour, J., \& Paz, S. (2004). Pain: Theories, evaluation and management. In S. Payne, J. Seymour, \& C. Ingleton. Palliative care nursing: Principles and evidence for practice, (pp. 260-298). London: Open University Press.

Silva, E. A., Neto, J. L., Figueiredo, M. C., \& Barbosa-Branco, A. (2007). Práticas e condutas que aliviam a dor e o sofrimento em crianças hospitalizadas. Comunicação em Ciências da Saúde, 18(2), 157-166. 
Silver, J. K. (2004). Chronic pain and the family. A new guide. Massachussets: Harvard University Press.

Simões, M. (2000). Investigação no âmbito da aferição nacional do teste das Matrizes Progressivas Coloridas de Raven (M.P.C.R.). Lisboa: Fundação Calouste Gulbenkian/ Fundação para a Ciência e Tecnologia.

Smith, A. A., \& Friedemann, M. (1999). Perceived family dynamics of persons with chronic pain. Journal of Advanced Nursing, 30(3), 543-551.

Spanier, G. B. (1976). Measuring dyadic adjustment: New scales for assessing the quality of marriage and similar dyads. Journal of Marriage and the Family, 38, 15-28.

West, C., Buettner, P., Foster, K., \& Usher, K. (2012). Pyschometric testing of the Family Impact of Pain Scale using a sample of families in Australia. Nurse Researcher, 20(2), 6-12.

West, C., Usher, K., Foster, K., \& Stewart, L. (2012). Chronic pain and the family: The experience of the partners of people living with chronic pain. Journal of clinical nursing, 21, 3352-3360. doi:10.1111/j.1365-2702.2012.04215.

Wilmut, J. (1975). Objective test analysis: Some criteria for item selection. Research in Education, 13, 27-56. 